\title{
Post-keratoplasty astigmatism management by relaxing incisions: a systematic review
}

\author{
Gaëlle Ho Wang Yin ${ }^{1,2,3^{*}}$ (D) and Louis Hoffart ${ }^{1,4}$
}

\begin{abstract}
Postoperative visual acuity can be limited by post-keratoplasty astigmatism, even with a clear corneal graft. Astigmatism management can be performed by selective suture removal, adjustment of sutures, optical correction, photorefractive procedures, wedge resection, intra-ocular lens implantation, intracorneal ring segments, relaxing incisions with or without compression sutures and repeated keratoplasty. Relaxing incisions can be made in the graft, graft-host interface or host cornea. Despite the unpredictability of the method because the flat and steep meridians are usually not orthogonal after penetrating keratoplasty, with asymmetric power distribution, all the studies showed an overall reduction of refractive, keratometric or topographic astigmatism, ranging from 30\% to $72 \%$ with manual or femtosecond-assisted techniques. Most patients with astigmatism higher than 6 diopters had residual cylinder less than or equal to 3 diopters, which can be treated by laser excimer ablation or secondary intraocular lens implantation.
\end{abstract}

Keywords: Penetrating keratoplasty, Astigmatic keratotomy, Relaxing incisions, Femtosecond laser

\section{Background}

The aim of penetrating keratoplasty (PK) is to improve a patient's postoperative vision. Despite a clear corneal graft, visual acuity can be limited by astigmatism [1]. Based on several studies, $15-20 \%$ of patients may develop 5 or more diopters (D) of post-keratoplasty astigmatism [2-5].

Post-keratoplasty astigmatism management can be done before or after suture removal. If done before, it is called suture-in post-keratoplasty astigmatism. In this case, topography-guided suture manipulation, including selective suture removal [6] guided by corneal topography [7], or adjustment of sutures along the steep meridian of astigmatism or adjustment of running sutures are key factors for controlling astigmatism.

After suture removal, post-keratoplasty astigmatism can be managed by optical correction such as contact lenses or glasses [8], which can be compromised by

\footnotetext{
* Correspondence: gaelle.ho-wang-yin@ap-hm.fr; gaelle-

howangyin@hotmail.fr

${ }^{1}$ Ophthalmology Department, Aix-Marseille University - APHM, Hôpital de la Timone, 264 rue Saint Pierre, 13385 Marseille Cedex 5, France

${ }^{2}$ Institut Fresnel UMR 7249, Aix Marseille Université, CNRS, Centrale Marseille,

Domaine universitaire de Saint-Jérôme Avenue Escadrille Normandie

Niemen, 13397 Marseille cedex 20, France

Full list of author information is available at the end of the article
}

irregular astigmatism associated with high-order aberrations [9]. Other astigmatism managements include incisional keratotomy in the graft $[10,11]$ or host cornea [12], and photorefractive procedures such as photorefractive keratectomy (PRK), laser in situ keratomileusis (LASIK) or topography-guided excimer laser ablation [13-16], compression sutures and a combination of relaxing incisions and compression sutures (augmented relaxing incisions) [17-19]. Wedge resection [20], intraocular lens (IOL) implantation [21] or intra-corneal ring segments (ICRS) [22] can also be considered. As a last resort, repeated keratoplasty [23] may be necessary.

The purpose of this review is to provide surgeon updated information about post-keratoplasty astigmatism management with arcuate keratotomy and to help define a strategy for the correction of this postkeratoplasty astigmatism. Questions we intend to answer were: Is femtosecond laser-assisted relaxing incision better than conventional relaxing incision for the management of post-penetrating keratoplasty astigmatism? Which nomogram and which length and depth should we use for the correction? 


\section{Main text}

\section{Search strategy}

A review on PubMed and Cochrane was performed, analyzing all the publications from 1986 to 2017, concerning the topic of post-keratoplasty astigmatism management with keratotomies (keywords: arcuate keratotomy, astigmatic keratotomy, penetrating keratoplasty, postkeratoplasty astigmatism). Clinical trials, systematic review, non-randomized prospective study and case series of more than 6 patients, published in an English language journal with an impact factor greater than or equal to 2.0 were reviewed.

\section{General considerations}

The corneal graft-host junction generally heals by 1 year after transplantation and corneal surface stability is generally achieved by 3 to 4 months after complete suture removal [24]. Before any surgical astigmatism management, a complete ophthalmologic examination including manifest and objective refraction, keratometry, and slit biomicroscopy should be performed to evaluate the graft-host interface and clarity of the graft, corneal topography, pachymetry, specular microscopy and wavefront analysis.

\section{Etiology of astigmatism}

Factors influencing post-penetrating keratoplasty astigmatism involve the donor tissue and the host.

Infant donor corneas induce greater astigmatism than adult donor tissues [25]. Non-uniform peripheral changes in the donor tissue can affect the apposition and healing between the host and donor tissue [26].

The severity of the underlying disorder (such as keratoconus, keratoglobus or pellucid marginal degeneration) [27] also plays a role in post-penetrating keratoplasty astigmatism.

During surgery, trephination (oval or eccentric) [28]; graft size (large graft diameters induce less astigmatism but are associated with a high risk for vascularization and allograft rejection, small grafts are associated with larger amounts of astigmatism) [29]; corneal thickness and donor-recipient disparity [30]; a poor suturing technique (single or double running sutures, interrupted sutures, all 3 techniques are comparable) [31] are factors involved in post-keratoplasty astigmatism. Time of suture removal or adjustment [32-34] is also important.

Post-operative drugs, inflammation, corneal vascularization, rejection and wound dehiscence affect wound healing and may be associated with astigmatic changes.

\section{Principles of relaxing incisions}

Relaxing incisions can be made for patients with keratometric astigmatism, 3 to 4 months after complete suture removal [24]. The main principle of relaxing incisions is to flatten the steep corneal meridian by one or two incisions perpendicular to it. This flattens the steepest meridian with reciprocal steepening of the meridian $90^{\circ}$ away, this effect is known as "coupling effect" [35]. Arc length is related to the coupling ratio: a coupling ratio of close to 1.0 is obtained with $30-90^{\circ}$ incisions, which should not change the resulting spherical equivalent. Incisions less than $20^{\circ}$ arc length have a coupling ratio greater than 1.0 while those greater than $100^{\circ}$ have a coupling ratio less than 1.0. Relaxing incisions are made under topical anesthesia, on both sides of the steepest meridian, usually, with an arc length of $45^{\circ}$ to $90^{\circ}$, and can be done in the $7.0 \mathrm{~mm}$ optical zone for maximal effect. The site and length are topography-guided [36]. In arcuate incisions, each incision is at the same distance from the visual axis to avoid uneven distribution of the force on the corneal architecture and allows rapid visual rehabilitation. Arcuate keratotomy can be made with or without 10-0 nylon compression sutures, these are added to achieve overcorrection of astigmatism in the opposite meridian ( $90^{\circ}$ away). Selective suture removal is initiated 3 to 4 weeks later.

The site of arcuate keratotomy can either be in the donor cornea or at the graft-host interface, or in the host cornea [12]. Incisions in the recipient corneas are not recommended as it is believed that the scarring at the graft-host junction changes the biomechanical state of the cornea. The keratoplasty wound is supposed to form a new limbus, blocking the effect of relaxing incisions in the recipient cornea [37].

Relaxing incisions can be made manually (freehand with a diamond knife [35] or mechanized with Hanna arcitome [38]) or with femtosecond laser technology [39].

\section{Nomograms}

The optical zone, arc length and depth are determined using nomograms. The effect of astigmatic correction with astigmatic keratotomy increases with greater incision depth, more central placement of the incisions, increasing patient age [40] and longer arc length.

Nomograms used on patients with congenital astigmatisms, such as the Lindstrom nomogram [40] do not apply to post-penetrating keratoplasty patients. The astigmatic effect of the incision is proportional to the preoperative cylinder [35]. Typically, post-penetrating keratoplasty arcuate keratotomy is made to a depth of $75 \%$ corneal thickness, and incisions are made at an optical zone $1.0 \mathrm{~mm}$ central to the graft-host junction.

The Hanna nomogram is one of the most widely used nomogram [38]. It is based on refractive astigmatism, from $2.50 \mathrm{D}$ to $15 \mathrm{D}$. The arcuate keratotomies are made with an optical zone diameter ranging from 6.00- 
$6.75 \mathrm{~mm}$, with an incision depth of $75 \%$ of corneal pachymetry and an angular length from 60 to $80^{\circ}$.

The Nordan nomogram [41] is used by surgeons to create paired symmetric incisions, $75-85 \%$ depth of the thinnest measurement of the graft, and centered on the steepest meridian axis as follows:

- 1.75-2.5 D of cylinder with $50^{\circ}$ arc length,

- 2.75-3.3 D of cylinder with $57^{\circ}$ arc length,

- 3.75-4.5 D of cylinder with $60^{\circ}$ arc length,

- $5 \mathrm{D}$ of astigmatism with $70^{\circ}$ arc length.

Another nomogram, for beveled incisions, was described by Cleary et al. [42]. These incisions, made at a $135^{\circ}$ angle centered on the axis of the astigmatism, allowed for the anterior cornea to slide forward, decreasing wound gape. Moreover, the beveled incisions can be made at a $65-75 \%$ depth rather than $90 \%$, with a reported comparable reduction in astigmatism versus traditional femtosecond laser-assisted arcuate keratotomy (FSAK) incisions. Arc length at $8.0 \mathrm{~mm}$ optical zone ranges from $60^{\circ}$ to $85^{\circ}$.

The most recent nomogram was developed by Saint-Clair [43]. It is a new nomogram for femtosecond laser astigmatic keratotomy for astigmatism after keratoplasty, considering a variety of incision-related factors and the degree of preexisting astigmatism. Incision depth, arc length and optical zone diameter depend on the pre-operative difference between the steepest and the flattest keratometry values.

\section{Manual versus femtosecond laser-assisted arcuate keratotomy}

Astigmatic keratotomy can be performed manually using a handheld, fixed or adjustable depth diamond knife, or mechanized like the Hanna arcitome. The diamond blade is adjusted according to the proper nomogram.

FSAK improve arc length, depth and location precision compared with manual and mechanized incision [39, 44]. The surgeon can adjust location, depth and centration of the incision using the intraoperative OCT of the femtosecond laser platform.

FSAK is also associated with a lower risk of wound dehiscence, epithelial down growth, infection, and perforation or full-thickness corneal incisions [39]. Main results are summarized further.

\section{Other techniques}

\section{Intrastromal astigmatic keratotomy}

Intrastromal astigmatic keratotomy (ISAK) was introduced for the treatment of low astigmatism. These incisions are not opened anteriorly, decreasing the risk of epithelial disruption, infection or wound dehiscence. ISAK was successful for naturally occurring and post- cataract surgery astigmatism [45] and residual astigmatism after refractive surgery [46].

Viswanathan \& Kumar [47] treated a young keratoconic patient with over $11 \mathrm{D}$ of astigmatism in both eyes after suture removal from bilateral penetrating keratoplasties. Paired ISAK with a depth of $90 \%, 60 \mu \mathrm{m}$ anteriorly at a $90^{\circ}$ side cut was made. At 4 months follow-up, keratometric astigmatism was $4.1 \mathrm{D}$ in the right eye and $1.12 \mathrm{D}$ in the left eye, with a corresponding reduction of $65.5 \%$ and $89.42 \%$, respectively.

Wetterstrand et al. [48] reported the results of intrastromal relaxing incisions after penetrating keratoplasty of 16 patients. The incisions were made intra-stromally with a length of $90^{\circ}$, depth of $90 \%$ of corneal thickness, diameter zone of $6.0-7.0 \mathrm{~mm}$, and a safe zone of $90 \mu \mathrm{m}$ anteriorly. Refractive cylinders decreased from $6.8 \pm 2.2 \mathrm{D}$ to $3.7 \pm 1.7$ $\mathrm{D}$ at 3 months follow-up, with only one bulge in the temporal incision of one patient, treated with compression suture. No other adverse effect was found. This technique seems to be relatively safe and effective.

\section{DIAKIK}

Loriaut et al. [49] described a novel technique, combining deep ISAK performed under a LASIK flap, for the treatment of high natural occurring and postkeratoplasty astigmatism: deep intrastromal arcuate keratotomy with in situ keratomileusis (DIAKIK).

The first step is ISAK made at a depth of $75 \%$ in eyes with penetrating keratoplasty. A LASIK flap of $100 \mu \mathrm{m}$ was then made, lifted and ISAK were opened and the flap replaced. Laser excimer ablation can be made 1 month after for the correction of residual ametropia, after reopening of the flap.

This technique was performed in 9 patients. The mean preoperative refractive cylinder was $6.11 \pm 2.54 \mathrm{D}$, and the post-operative cylinder was $2.85 \pm 1.31 \mathrm{D}$. No complications were reported. This technique affords advantages of ISAK with a greater astigmatism correction, but there are potential complications of the LASIK itself.

\section{Results}

Main results are summarized in Table 1.

Arcuate keratotomy can be useful as it creates minimal surface disruption, can treat nonorthogonal astigmatism, and yields rapid visual recovery. This technique ensures an optical zone of constant diameter and homogeneity of depth over the entire length of the incision. Typically, post-PK keratotomies are done to a depth of $75 \%$ to $80 \%$ corneal thickness, and incisions are made at an optical zone $1.0 \mathrm{~mm}$ central to the graft-host junction.

Three different incision locations were described: graft, graft-host interface or host cornea. Refractive or keratometric pre-operative cylinder was between $11 \mathrm{D}$ and 6.1 $\mathrm{D}$, and an overall reduction ranging from 30 to $72 \%$ was 
Table 1 Main results studies

\begin{tabular}{|c|c|c|c|c|c|c|c|c|c|}
\hline \multirow[t]{2}{*}{ Authors } & \multirow[t]{2}{*}{$\mathrm{N}$} & \multirow[t]{2}{*}{ Study type } & \multirow{2}{*}{\multicolumn{2}{|c|}{ Treatment }} & \multirow[t]{2}{*}{ Location } & \multicolumn{3}{|c|}{$\begin{array}{l}\text { Refractive }(R) \text { or } \\
\text { keratometric }(K) \text { cylinder }\end{array}$} & \multirow[t]{2}{*}{ Complications } \\
\hline & & & & & & $\begin{array}{l}\text { Pre-op } \\
\text { (D) }\end{array}$ & $\begin{array}{l}\text { Post-op } \\
\text { (D) }\end{array}$ & $\begin{array}{l}\begin{array}{l}\text { Reduction } \\
(\%)\end{array} \\
\end{array}$ & \\
\hline $\begin{array}{l}\text { Krachmer \& Fenzl, } \\
1980 \text { [52] }\end{array}$ & 16 & $\begin{array}{l}\text { Retrospective, comparative } \\
\text { case series }\end{array}$ & \multicolumn{2}{|c|}{$\begin{array}{l}\text { Manual paired } \\
\text { relaxing incision } \\
\text { Depth }=50 \% \text { to } 66 \% \mathrm{CCT} \\
\text { Length }=60^{\circ} \\
\text { Graft-host interface }\end{array}$} & $\begin{array}{l}\text { Graft-host } \\
\text { interface }\end{array}$ & $10.11(\mathrm{~K})$ & $5.79(\mathrm{~K})$ & $43(\mathrm{~K})$ & 2 perforations \\
\hline $\begin{array}{l}\text { Price \& Whitson, } 1991 \\
\text { [55] }\end{array}$ & 111 & Retrospective case series & \multicolumn{2}{|c|}{$\begin{array}{l}\text { Manual paired } \\
\text { relaxing incision } \\
\text { Depth and length variable }\end{array}$} & $\begin{array}{l}\text { Graft or } \\
\text { graft-host } \\
\text { interface }\end{array}$ & $\begin{array}{l}6.18 \pm 1.56 \\
(\mathrm{R})\end{array}$ & $\begin{array}{l}3.34 \pm 1.96 \\
(\mathrm{R})\end{array}$ & $46(\mathrm{R})$ & $\begin{array}{l}12 \text { wound } \\
\text { dehiscences }\end{array}$ \\
\hline $\begin{array}{l}\text { Hjortdal \& Ehlers, } 1998 \\
\text { [56] }\end{array}$ & 21 & $\begin{array}{l}\text { Nonrandomized } \\
\text { retrospective study }\end{array}$ & \multicolumn{2}{|c|}{$\begin{array}{l}\text { Manual relaxing incision } \\
\text { Depth }=\text { central corneal thickness } \\
\text { Length }=45^{\circ} ; 5 \text { or } 6 \mathrm{~mm} \\
\text { from optical zone }\end{array}$} & Graft & $7(\mathrm{R})$ & 3.25 (R) & $54(\mathrm{R})$ & None \\
\hline $\begin{array}{l}\text { Hannush et al., } 1998 \\
\text { [57] }\end{array}$ & 29 & Retrospective case series & \multicolumn{2}{|c|}{$\begin{array}{l}\text { Manual paired relaxing incision } \\
\text { Depth }=75 \% \mathrm{CC} \\
\text { Length }=\text { not available } \\
0.5 \mathrm{~mm} \text { inside the } \\
\text { graft-host interface }\end{array}$} & Graft & $\begin{array}{l}8.8 \pm 3.1 \\
(\mathrm{~K})\end{array}$ & $\begin{array}{l}3.2 \pm 3.0 \\
(K)\end{array}$ & $64(K)$ & None \\
\hline \multirow[t]{2}{*}{$\begin{array}{l}\text { Borderie et al., } 1999 \\
\text { [58] }\end{array}$} & \multirow[t]{2}{*}{22} & \multirow[t]{2}{*}{ Retrospective case series } & \multirow{2}{*}{\multicolumn{2}{|c|}{$\begin{array}{l}\text { Manual paired relaxing } \\
\text { incision with Hanna arcitome } \\
\text { Depth }=75 \% \text { thinnest pachymetry } \\
\text { Length }=60^{\circ} \text { to } 80^{\circ} \\
6 \text { to } 6.75 \mathrm{~mm} \text { zone diameter } \\
\text { (Hanna nomogram [38]) }\end{array}$}} & Graft & $\begin{array}{l}6.94 \pm 2.11 \\
(\mathrm{R})\end{array}$ & $\begin{array}{l}3.85 \pm 1.95 \\
\text { (R) }\end{array}$ & $44(\mathrm{R})$ & \multirow{2}{*}{$\begin{array}{l}1 \text { perforation, } \\
1 \text { overcorrection } \\
\text { (same patient) }\end{array}$} \\
\hline & & & & & & $\begin{array}{l}9.02 \pm 2.67 \\
(K)\end{array}$ & $3.94 \pm 2.19(\mathrm{~K})$ & $56(\mathrm{~K})$ & \\
\hline \multirow[t]{2}{*}{$\begin{array}{l}\text { Koay et al., } \\
2000[59]\end{array}$} & \multirow[t]{2}{*}{34} & \multirow[t]{2}{*}{$\begin{array}{l}\text { Nonrandomized } \\
\text { retrospective study }\end{array}$} & \multirow{2}{*}{\multicolumn{2}{|c|}{$\begin{array}{l}\text { Manual relaxing incision and } \\
\text { compression sutures } \\
\text { Depth }=480 \mu \mathrm{m} \\
\text { Length }=45^{\circ}\end{array}$}} & $\begin{array}{l}\text { Graft-host } \\
\text { interface }\end{array}$ & $9.69 \pm 3.51(\mathrm{R})$ & $\begin{array}{l}3.92 \pm 2.16 \\
\text { (R) }\end{array}$ & $59.5(\mathrm{R})$ & \multirow[t]{2}{*}{1 graft rejection } \\
\hline & & & & & & $9.14 \pm 4.38(\mathrm{~K})$ & $\begin{array}{l}3.59 \pm 1.92 \\
(\mathrm{~K})\end{array}$ & $60.7(K)$ & \\
\hline Wilkins et al., 2005 [35] & 20 & $\begin{array}{l}\text { Nonrandomized } \\
\text { retrospective study }\end{array}$ & $\begin{array}{l}\text { Manual relaxing incisior } \\
\text { Depth }=600 \mu \mathrm{m} \\
\text { Length }=60^{\circ} ; 6 \mathrm{~mm} \text { fro } \\
\text { optical zone }\end{array}$ & & Host & $\begin{array}{l}10.99 \pm 4.26 \\
(\mathrm{R})\end{array}$ & $\begin{array}{l}3.33 \pm 2.18 \\
(\mathrm{R})\end{array}$ & $72(\mathrm{R})$ & None \\
\hline \multirow{2}{*}{$\begin{array}{l}\text { Bochmann \& Schipper, } \\
2006[12]\end{array}$} & \multirow[t]{2}{*}{11} & \multirow{2}{*}{$\begin{array}{l}\text { Nonrandomized } \\
\text { retrospective study }\end{array}$} & \multirow{2}{*}{\multicolumn{2}{|c|}{$\begin{array}{l}\text { Manual relaxing incision } \\
\text { Depth }=80 \% \text { of peripheral } \\
\text { corneal thickess } \\
\text { Length }=30^{\circ} \text { to } 90^{\circ} \\
9 \text { to } 10 \mathrm{~mm} \text { zone diameter }\end{array}$}} & Host & $6.1 \pm 2.5(\mathrm{R})$ & $3.3 \pm 0.7(\mathrm{R})$ & 45.9 (R) & \multirow[t]{2}{*}{ None } \\
\hline & & & & & & $9.02 \pm 3.54(\mathrm{~K})$ & $3.41 \pm 1.21(\mathrm{~K})$ & $62.2(\mathrm{~K})$ & \\
\hline Geggel 2006, [50] & 26 & $\begin{array}{l}\text { Nonrandomized } \\
\text { retrospective observational } \\
\text { case series }\end{array}$ & $\begin{array}{l}\text { Manual relaxing incisior } \\
\text { Depth }=80 \% \text { of the thi } \\
\text { pachymetry on the me } \\
\text { Length }=35^{\circ} \text { to } 90^{\circ} \\
0.5 \mathrm{~mm} \text { or } 1 \mathrm{~mm} \text { inside } \\
\text { graft-host junction if }>\end{array}$ & $\begin{array}{l}\text { on } \\
\text { innest } \\
\text { eridian } \\
\text { de the } \\
10 \mathrm{D}\end{array}$ & Graft & $\begin{array}{l}8.7 \pm 2.4 \\
(K)\end{array}$ & $3.25 \pm 1.74(\mathrm{~K})$ & $63(\mathrm{~K})$ & None \\
\hline $\begin{array}{l}\text { Poole \& Ficker, } \\
2006 \text { [60] }\end{array}$ & 39 & $\begin{array}{l}\text { Nonrandomized } \\
\text { retrospective study }\end{array}$ & $\begin{array}{l}\text { Manual relaxing incisior } \\
\text { Depth }=90 \% \mathrm{CC} \\
\text { Length }=45^{\circ} \text { to } 90^{\circ}, 0.5 \\
\text { inside the graft-host int }\end{array}$ & $\begin{array}{l}\text { on } \\
.5 \mathrm{~mm} \\
\text { nterface }\end{array}$ & Graft & $9.13(\mathrm{R})$ & $4.85(\mathrm{R})$ & $47(\mathrm{R})$ & 1 perforation \\
\hline Hoffart et al., 2007 [61] & 40 & $\begin{array}{l}\text { Nonrandomized } \\
\text { retrospective study }\end{array}$ & $\begin{array}{l}\text { Manual relaxing incisior } \\
\text { Depth }=75 \% \mathrm{CCT} \text {, } \\
\text { length }=60^{\circ} \text { to } 80^{\circ} \\
6 \text { to } 6.75 \mathrm{~mm} \text { zone dial } \\
\text { (Hanna nomogram [38. }\end{array}$ & 8]) & Graft & $\begin{array}{l}8.84 \pm 3 \\
\text { (R) }\end{array}$ & $4.88 \pm 2.5(\mathrm{R})$ & $45(\mathrm{R})$ & $\begin{array}{l}1 \text { perforation, } \\
2 \text { rejections }\end{array}$ \\
\hline \multirow[t]{2}{*}{ Bahar et al., 2008 [62] } & \multirow[t]{2}{*}{40} & \multirow[t]{2}{*}{$\begin{array}{l}\text { Retrospective, } \\
\text { comparative case series }\end{array}$} & \multirow[t]{2}{*}{$\begin{array}{l}\text { FS: Depth }=90 \% \mathrm{CCT} \\
\text { Length }=60^{\circ} \text { to } 90^{\circ} \\
0.5 \mathrm{~mm} \text { inside the } \\
\text { graft-host junction }\end{array}$} & \multirow[t]{2}{*}{$\begin{array}{l}\text { Manual: Depth }=500 \mu \mathrm{m} \\
\text { Length }=45^{\circ} \text { to } 90^{\circ} \\
0.5 \mathrm{~mm} \text { inside the } \\
\text { graft-host junction }\end{array}$} & \multirow[t]{2}{*}{ Graft } & $\begin{array}{l}\text { FS: } 7.84 \pm 2.35 \\
(\mathrm{~K})\end{array}$ & $\begin{array}{l}\text { FS: } 3.58 \pm \\
2.21(\mathrm{~K})\end{array}$ & FS: $54(\mathrm{~K})$ & $\begin{array}{l}\text { FS: } \\
5 \text { overcorrections, } \\
1 \text { rejection }\end{array}$ \\
\hline & & & & & & $\begin{array}{l}\text { Manual: } \\
7.80 \pm 3.14(\mathrm{~K})\end{array}$ & $\begin{array}{l}\text { Manual: } \\
4.58 \pm 2.95(\mathrm{~K})\end{array}$ & $\begin{array}{l}\text { Manual: } \\
41(K)\end{array}$ & $\begin{array}{l}3 \text { perforations, } \\
6 \text { overcorrections, } \\
1 \text { infection }\end{array}$ \\
\hline \multirow[t]{2}{*}{$\begin{array}{l}\text { Buzzonetti et al., } \\
2009 \text { [63] }\end{array}$} & 9 & $\begin{array}{l}\text { Prospective non- } \\
\text { comparative study }\end{array}$ & $\begin{array}{l}\text { FS, paired symmetric in } \\
\text { Depth }=80 \% \text { thinnest }\end{array}$ & $\begin{array}{l}\text { incisions } 90^{\circ} \text { angled } \\
\text { pachymetry }\end{array}$ & Graft & $9.10 \pm 3.90(\mathrm{R})$ & $\begin{array}{l}3.10 \pm 1.50 \\
(R)\end{array}$ & $66(\mathrm{R})$ & None \\
\hline & & & $\begin{array}{l}\text { Length }=10^{\circ} \\
\text { Optical zone }=4.8 \text { to } 6 \text {. }\end{array}$ & $6.8 \mathrm{~mm}$ & & $9.80 \pm 1.90(\mathrm{~K})$ & $\begin{array}{l}5.20 \pm 1.50 \\
(\mathrm{~K})\end{array}$ & $47(K)$ & \\
\hline Nubile et al., 2009 [64] & 12 & $\begin{array}{l}\text { Prospective } \\
\text { noncomparative } \\
\text { interventional case series. }\end{array}$ & $\begin{array}{l}\text { FS, paired symmetric in } \\
\text { Depth }=90 \% \text { pachymet } \\
\text { Length }=40^{\circ} \text { to } 80^{\circ} \\
1 \mathrm{~mm} \text { inside the graft- }\end{array}$ & $\begin{array}{l}\text { incisions } 90^{\circ} \text { angled } \\
\text { etry on incision location } \\
\text {-host junction }\end{array}$ & Graft & $7.16 \pm 3.07(\mathrm{R})$ & $\begin{array}{l}2.39 \pm 1.62 \\
(R)\end{array}$ & $66(R)$ & 2 perforations \\
\hline Hoffart et al., 2009 [39] & 20 & $\begin{array}{l}\text { Prospective comparative } \\
\text { randomized study }\end{array}$ & $\begin{array}{l}\text { FS or Hanna arcitome } \\
\text { Depth }=75 \% \text { thinnest p }\end{array}$ & pachymetry & Graft & $\mathrm{FS}: 8.6 \pm 3(\mathrm{R})$ & $\begin{array}{l}\text { FS: } 3.9 \pm 2.4 \\
\text { (R) }\end{array}$ & FS: 55 (R) & 1 perforation \\
\hline
\end{tabular}


Table 1 Main results studies (Continued)

\begin{tabular}{|c|c|c|c|c|c|c|c|c|}
\hline \multirow[t]{2}{*}{ Authors } & \multirow[t]{2}{*}{ N } & \multirow[t]{2}{*}{ Study type } & \multirow[t]{2}{*}{ Treatment } & \multirow[t]{2}{*}{ Location } & \multicolumn{3}{|c|}{$\begin{array}{l}\text { Refractive (R) or } \\
\text { keratometric (K) cylinder }\end{array}$} & \multirow[t]{2}{*}{ Complications } \\
\hline & & & & & $\begin{array}{l}\text { Pre-op } \\
\text { (D) }\end{array}$ & $\begin{array}{l}\text { Post-op } \\
\text { (D) }\end{array}$ & $\begin{array}{l}\text { Reduction } \\
(\%)\end{array}$ & \\
\hline & & & $\begin{array}{l}\text { Length }=60^{\circ} \text { to } 80^{\circ} \\
6 \text { to } 6.75 \mathrm{~mm} \text { zone diameter } \\
\text { (Hanna nomogram [38]) }\end{array}$ & & $\begin{array}{l}\text { Hanna: } \\
6.7 \pm 2.1(\mathrm{R})\end{array}$ & $\begin{array}{l}\text { Hanna: } \\
4.7 \pm 2.4 \text { (R) }\end{array}$ & $\begin{array}{l}\text { Hanna: } \\
30(\mathrm{R})\end{array}$ & \\
\hline Kumar et al. 2010 [65] & 34 & Retrospective case series & $\begin{array}{l}\text { FS, paired symmetric incisions } \\
\text { Depth }=90 \% \text { pachymetry on incision location } \\
\text { Length }=40^{\circ} \text { to } 60^{\circ}(<6 \mathrm{D}), 65^{\circ} \text { to } 75^{\circ} \\
(6 \text { to } 10 \mathrm{D}), 90^{\circ}(>10 \mathrm{D}) \\
0.5 \mathrm{~mm} \text { inside the graft-host junction }\end{array}$ & Graft & $7.46 \pm 2.7(K)$ & $4.77 \pm 3.29(\mathrm{~K})$ & $36(\mathrm{~K})$ & $\begin{array}{l}3 \text { rejections, } \\
9 \text { overcorrections } \\
\text { (24\%) }\end{array}$ \\
\hline Fares et al., 2013 [66] & 26 & $\begin{array}{l}\text { Nonrandomized } \\
\text { prospective study }\end{array}$ & $\begin{array}{l}\text { Manual relaxing incision } \\
\text { with compression suture } \\
\text { Depth }=80 \% \mathrm{CC} \\
\left.\text { Length }=45^{\circ} \text { ( } 6 \mathrm{D} \text { to } 9 \mathrm{D}\right) \text { to } 60^{\circ}(>9 \mathrm{D}) \\
7 \mathrm{~mm} \text { zone diameter }\end{array}$ & Interface & $9.66 \pm 2.9(\mathrm{R})$ & $\begin{array}{l}4.37 \pm 2.53 \\
\text { (R) }\end{array}$ & $58.4(\mathrm{R})$ & 1 perforation \\
\hline Cleary et al., 2013 [42] & 6 & $\begin{array}{l}\text { Prospective } \\
\text { noncomparative } \\
\text { interventional case series }\end{array}$ & $\begin{array}{l}\text { FS beveled } \\
\text { Bevel angle of } 135^{\circ} \\
\text { Depth }=65 \% \text { to } 75 \% \mathrm{CCT} \\
\text { Length }=60^{\circ} \text { to } 90^{\circ} \\
0.4 \mathrm{~mm} \text { inside the graft margin }\end{array}$ & Graft & $9.8 \pm 2.9(\mathrm{~K})$ & $4.5 \pm 3.2(\mathrm{~K})$ & $57(\mathrm{~K})$ & None \\
\hline Loriaut et al., 2015 [67] & 20 & Retrospective case series & $\begin{array}{l}\text { FS } \\
\text { Depth }=75 \% \text { thinnest pachymetry } \\
\text { Length }=60^{\circ} \text { to } 80^{\circ} \\
6 \text { to } 6.8 \mathrm{~mm} \text { zone diameter (Hanna nomogram } \\
{[38] \text { ) }}\end{array}$ & Graft & $9.45 \pm 2.97(\mathrm{~K})$ & $4.64 \pm 2.79(\mathrm{~K})$ & $50.9(\mathrm{~K})$ & 10 overcorrections \\
\hline $\begin{array}{l}\text { Al Sabaani et al., } \\
2016 \text { [41] }\end{array}$ & 52 & $\begin{array}{l}\text { Retrospective } \\
\text { non-comparative } \\
\text { interventional study }\end{array}$ & $\begin{array}{l}\text { FS } \\
\text { Depth }=75 \%-85 \% \text { thinnest pachymetry } \\
\text { Length }=50^{\circ} \text { to } 70^{\circ} \text { (Nordan nomogram [41]) } \\
0.5-0.7 \mathrm{~mm} \text { within the graft-host junction }\end{array}$ & Graft & $7.15 \pm 1.32(\mathrm{R})$ & $\begin{array}{l}5.19 \pm 2.25 \\
(\mathrm{R})\end{array}$ & 27.4 (R) & $\begin{array}{l}3 \text { perforations, } \\
12 \text { overcorrections }\end{array}$ \\
\hline $\begin{array}{l}\text { Hashemian et al., } \\
2017 \text { [68] }\end{array}$ & 23 & $\begin{array}{l}\text { Prospective interventional } \\
\text { case series }\end{array}$ & $\begin{array}{l}\text { FS } \\
\text { Depth }=85 \% \text { thinnest pachymetry } \\
\text { Length }=40^{\circ} \text { to } 90^{\circ} \text { (Wu [69] and Kumar [65] } \\
\text { nomogram) } \\
1 \mathrm{~mm} \text { within the graft-host junction }\end{array}$ & Graft & $7.79 \pm 2.64$ (R) & $\begin{array}{l}3.69 \pm 2.25 \\
(\mathrm{R})\end{array}$ & $52.6(\mathrm{R})$ & 2 perforations \\
\hline
\end{tabular}

$C C T=$ central corneal thickness; $F S=$ femtosecond laser assisted

observed, with manual or femtosecond-assisted techniques. Most of the patients with astigmatism higher than $6 \mathrm{D}$ had residual cylinder less or equal to $3 \mathrm{D}$, which can be treated by laser excimer ablation, or secondary IOL implantation.

In the reported studies, manual keratotomy seems to have more complications (in particular wound dehiscence) and poorer predictability than FSAK. However, all studies had a high variability in outcomes despite the use of a standardized method of surgery. The unpredictability seems to be correlated with many variables such as the value of the initial cylinder, individual variability, alignment.

Individual variability responsible for limited predictability is related to the variability in the distribution of compressive forces and biomechanical constraints in the corneal graft. Therefore, arcuate keratotomy after PK necessitates its own nomogram, as the fibrotic rim created at the graft-host junction has a different tension than the natural limbus.

Under-correction and inter-individual variability can be due to misalignment. This could be addressed by preoperative limbal marking but the direct visualization of the limbus can be masked by the suction ring during the procedure. Also, a control of cyclotorsion can be useful in this case. Femtosecond provides more depth accuracy than manual keratotomy as we have a preoperative view of the cornea thickness thanks to the OCT.

Unsuccessful correction and overcorrection can be explained by the pattern of irregular astigmatism (the flat and steep meridians are usually not orthogonal, with asymmetric power distribution), which leads to unpredictable correction. Some authors hypothesized that using topography-guided placement of relaxing incision can produce more predictable results, as shown by vector analysis using Alpin's methods [39, 50, 51].

Unpredictability can also be explained by the inherent dynamic instability of such incisions over time.

It is possible that a smaller arc length would achieve the intended effect on astigmatism or increasing the angular length and reducing the optical zone diameter of the incisions as Wilkins et al. [35] reported.

A refinement in the treatment nomogram for femtosecond laser-assisted AK for high astigmatism after PK remains a major issue. Further prospective studies with different length, depth and width correlated with the pre-operative cylinder and optical zone should be performed to define a new nomogram with a higher predictability. 


\section{Complications}

Manual procedures have some disadvantages: poor predictability, overcorrection, corneal perforation, wound dehiscence, and instability of corneal topography [24]. In manual procedures, depth is usually set to $75-85 \%$ depth of central corneal thickness (CCT), but preoperative visualization of the corneal thickness is not possible thereby increasing the risk of perforation and overcorrection due to deep incisions. Deep incisions are also associated with wound dehiscence. The suture of the keratotomy can reduce dehiscence and overcorrection, and can be used to treat micro-perforation. Poor predictability with manual procedures is due to the absence of standardized nomograms correlating the amount of keratometric astigmatism with the extension of the incisions. Nomogram for congenital astigmatism cannot be applied for post-PK astigmatism [52], so newer and more precise nomogram should be studied.

FSAK is safer and more predictable than manual procedures, but complications have also been described (Table 1). Overcorrection or wound gaping can be managed with compressive sutures. Infections are rare but possible, keratitis and one case of endophthalmitis have been reported. Topical post-operative antibioprophylaxis should be sufficient [53]. Graft rejections have also been reported, but topical corticosteroid for 1 month after the surgery can be administered. Corneal perforation can occur, even with per-operative OCT [54], but it is a very rare complication.

\section{Conclusion}

No standard surgical procedure for performing arcuate keratotomies for post-penetrating keratoplasties astigmatism exists, due to its unpredictability. However, all the studies show that patients with astigmatism higher than $6 \mathrm{D}$ had residual cylinder less than or equal to $3 \mathrm{D}$, which can be further treated by laser excimer ablation or secondary IOL implantation.

\section{Acknowledgements}

Not applicable.

\section{Funding}

Not applicable.

\section{Availability of data and materials}

Data sharing is not applicable to this article as no datasets were generated or analyzed during the current study.

\section{Authors' contributions}

$\mathrm{LH}$ and $\mathrm{GH}$ made substantial contributions to conception and design, and interpretation of data of the review; have been involved in drafting the manuscript or revising it critically for important intellectual content. All the authors gave final approval of the version to be published.

Ethics approval and consent to participate Not applicable.
Consent for publication

Not applicable.

\section{Competing interests}

The authors declare that they have no competing interests.

\section{Author details}

'Ophthalmology Department, Aix-Marseille University - APHM, Hôpital de la Timone, 264 rue Saint Pierre, 13385 Marseille Cedex 5, France. ${ }^{2}$ Institut Fresnel UMR 7249, Aix Marseille Université, CNRS, Centrale Marseille, Domaine universitaire de Saint-Jérôme Avenue Escadrille Normandie Niemen, 13397 Marseille cedex 20, France. ${ }^{3}$ CERIMED, Aix-Marseille University, 27 Boulevard Jean-Moulin, 13385 Marseille cedex 05, France. ${ }^{4}$ Ramsay Générale de Santé, Clinique Monticelli-Velodrome, Marseille, France.

Received: 27 August 2017 Accepted: 26 November 2017

Published online: 06 December 2017

\section{References}

1. Williams KA, Hornsby NB, Bartlett CM, et al. Report from the Australian corneal graft registry. Tech. Rep. Snap Printing: Adelaide; 2004.

2. Binder PS. The effect of suture removal on postkeratoplasty astigmatism. Am J Ophthalmol. 1988;105:637-45.

3. Limberg MB, Dingeldein SA, Green MT, Klyce SD, Insler MS, Kaufman HE. Corneal compression sutures for the reduction of astigmatism after penetrating keratoplasty. Am J Ophthalmol. 1989;108:36-42.

4. Swinger CA. Postoperative astigmatism. Surv Ophthalmol. 1987;31:219-48.

5. Troutman RC, Lawless MA. Penetrating keratoplasty for keratoconus. Cornea. 1987;6:298-305.

6. Binder PS. The effect of suture removal on postkeratoplasty astigmatism [letter]. Am J Ophthalmol. 1988;106:507.

7. Sarhan AR, Dua HS, Beach M. Effect of disagreement between refractive, keratometric, and topographic determination of astigmatic axis on suture removal after penetrating keratoplasty. Br J Ophthalmol. 2000;84:837-41.

8. Price FW Jr, Whitson WE, Marks RG. Progression of visual acuity after penetrating keratoplasty. Ophthalmology. 1991;98:1177-85.

9. Rajan MS, O'Brart DP, Patel P, Falcon MG, Marshall J. Topography-guided customized laser-assisted subepithelial keratectomy for the treatment of postkeratoplasty astigmatism. J Cataract Refract Surg. 2006;32:949-57.

10. Fronterrè A, Portesani GP. Relaxing incisions for postkeratoplasty astigmatism. Cornea. 1991;10:305-11.

11. Høvding G. Transverse keratotomy in postkeratoplasty astigmatism. Acta Ophthalmol (Copenh). 1994;72:464-8.

12. Bochmann F, Schipper I. Correction of post-keratoplasty astigmatism with keratotomies in the host cornea. J Cataract Refract Surg. 2006;32:923-8.

13. Tuunanen TH, Ruusuvaara PJ, Uusitalo RJ, Tervo TM. Photoastigmatic keratectomy for correction of astigmatism in corneal grafts. Cornea. 1997;16:48-53.

14. Kirkness CM, Ficker LA, Steele AD, Rice NS. Refractive surgery for graftinduced astigmatism after penetrating keratoplasty for keratoconus. Ophthalmology. 1991;98:1786-92.

15. Lazzaro DR, Haight DH, Belmont SC, Gibralter RP, Aslanides IM, Odrich MG. Excimer laser keratectomy for astigmatism occurring after penetrating keratoplasty. Ophthalmology. 1996;103:458-64.

16. Lavery GW, Lindstrom RL, Hofer LA, Doughman DJ. The surgical management of corneal astigmatism after penetrating keratoplasty. Ophthalmic Surg. 1985;16:165-9.

17. Mandel MR, Shapiro MB, Krachmer JH. Relaxing incisions with augmentation sutures for the correction of postkeratoplasty astigmatism. Am J Ophthalmol. 1987;103:441-7.

18. McCartney DL, Whitney CE, Stark WJ, Wong SK, Bernitsky DA. Refractive keratoplasty for disabling astigmatism after penetrating keratoplasty. Arch Ophthalmol. 1987;105:954-7.

19. Javadi MA, Feizi S, Yazdani S, Sharifi A, Sajjadi H. Outcomes of augmented relaxing incisions for postpenetrating keratoplasty astigmatism in keratoconus. Cornea. 2009;28:280-4.

20. Lugo M, Donnenfeld ED, Arentsen JJ. Corneal wedge resection for high astigmatism following penetrating keratoplasty. Ophthalmic Surg. 1987;18:650-3.

21. Viestenz A, Küchle M, Seitz B, Langenbucher A. Toric intraocular lenses for correction of persistent corneal astigmatism after penetrating keratoplasty. Ophthalmologe. 2005;102:148-52. 
22. Arriola-Villalobos P, Díaz-Valle D, Güell JL, Iradier-Urrutia MT, Jiménez-Alfaro I, Cuiña-Sardiña R, et al. Intrastromal corneal ring segment implantation for high astigmatism after penetrating keratoplasty. J Cataract Refract Surg. 2009:35:1878-84.

23. Szentmáry N, Seitz B, Langenbucher A, Naumann GO. Repeat keratoplasty for correction of high or irregular postkeratoplasty astigmatism in clear corneal grafts. Am J Ophthalmol. 2005;139:826-30.

24. Feizi S, Zare M. Current Approaches for Management of Postpenetrating Keratoplasty Astigmatism. J Ophthalmol. 2011;2011:708736.

25. Palay DA, Kangas TA, Stulting RD, Winchester K, Litoff D, Krachmer JH. The effects of donor age on the outcome of penetrating keratoplasty in adults. Ophthalmology. 1997;104:1576-9.

26. Butrus SI, Ashraf MF, Azar DT. Postkeratoplasty astigmatism: etiology, management and femtosecond laser applications. In: Azar DT, editor. Refractive surgery. 2nd ed. St. Louis: Mosby-Elsevier; 2007. p. 549-59.

27. Riddle HK Jr, Parker DA, Price FW Jr. Management of postkeratoplasty astigmatism. Curr Opin Ophthalmol. 1998;9:15-28.

28. Cohen $\mathrm{KL}$, Holman RE, Tripoli NK, Kupper LL. Effect of trephine tilt on corneal button dimensions. Am J Ophthalmol. 1986;101:722-5.

29. Seitz B, Langenbucher A, Küchle M, Naumann GO. Impact of graft diameter on corneal power and the regularity of postkeratoplasty astigmatism before and after suture removal. Ophthalmology. 2003;110:2162-7.

30. Woodford SV. Control of postkeratoplasty astigmatism. In: Brightbill FS, editor. Corneal surgery: theory, technique and tissue. 3rd ed. New York: Mosby; 1999. p. 431-40.

31. Javadi MA, Naderi M, Zare M, Jenaban A, Rabei HM, Anissian A. Comparison of the effect of three suturing techniques on postkeratoplasty astigmatism in keratoconus. Cornea. 2006;25:1029-33.

32. Karabatsas $\mathrm{CH}$, Cook SD, Figueiredo FC, Diamond JP, Easty DL. Combined interrupted and continuous versus single continuous adjustable suturing in penetrating keratoplasty: a prospective, randomized study of induced astigmatism during the first postoperative year. Ophthalmology. 1998;105:1991-8.

33. Burk LL, Waring GO 3rd, Radjee B, Stulting RD. The effect of selective suture removal on astigmatism following penetrating keratoplasty. Ophthalmic Surg. 1988;19:849-54.

34. Musch DC, Meyer RF, Sugar A. The effect of removing running sutures on astigmatism after penetrating keratoplasty. Arch Ophthalmol. 1988;106:488-92.

35. Wilkins MR, Mehta JS, Larkin DF. Standardized arcuate keratotomy for postkeratoplasty astigmatism. J Cataract Refract Surg. 2005;31:297-301.

36. Feizi S. Management of Post-Penetrating Keratoplasty Astigmatism. In: Goggin M, editor. Astigmatism - optics, physiology and management [Internet], InTech; 2012.

37. Roberts C. The cornea is not a piece of plastic. J Refract Surg. 2000;16:407-13.

38. Hanna KD, Hayward JM, Hagen KB, Simon G, Parel JM, Waring GO 3rd. Keratotomy for astigmatism using an arcuate keratome. Arch Ophthalmol. 1993;111:998-1004.

39. Hoffart L, Proust H, Matonti F, Conrath J, Ridings B. Correction of postkeratoplasty astigmatism by femtosecond laser compared with mechanized astigmatic keratotomy. Am J Ophthalmol. 2009;147:779-87.

40. Price FW, Grene RB, Marks RG, Gonzales JS. Astigmatism reduction clinical trial: a multicenter prospective evaluation of the predictability of arcuate keratotomy. Evaluation of surgical nomogram predictability. ARC-T Study Group. Arch Ophthalmol. 1995;113:277-82.

41. Al Sabaani N, Al Malki S, Al Jindan M, Al Assiri A, Al Swailem S. Femtosecond astigmatic keratotomy for postkeratoplasty astigmatism. Saudi J Ophthalmol. 2016;30:163-8.

42. Cleary C, Tang M, Ahmed H, Fox M, Huang D. Beveled femtosecond laser astigmatic keratotomy for the treatment of high astigmatism postpenetrating keratoplasty. Cornea. 2013;32:54-62.

43. St Clair RM, Sharma A, Huang D, Yu F, Goldich Y, Rootman D, et al. Development of a nomogram for femtosecond laser astigmatic keratotomy for astigmatism after keratoplasty. J Cataract Refract Surg. 2016;42:556-62.

44. Hurmeric V, Yoo SH. Femtosecond-assisted astigmatic keratotomy. Cataract Refract Surg Today Europe. 2009:30-3.

45. Rückl T, Dexl AK, Bachernegg A, Reischl V, Riha W, Ruckhofer J, et al. Femtosecond laser-assisted intrastromal arcuate keratotomy to reduce corneal astigmatism. J Cataract Refract Surg. 2013;39:528-38. http://www. crstodayeurope.com.

46. Venter J, Blumenfeld R, Schallhorn S, Pelouskova M. Non-penetrating femtosecond laser intrastromal astigmatic keratotomy in patients with mixed astigmatism after previous refractive surgery. J Refract Surg. 2013;29:180-6.
47. Viswanathan D, Kumar NL. Bilateral femtosecond laser-enabled intrastromal astigmatic keratotomy to correct high post-penetrating keratoplasty astigmatism. J Cataract Refract Surg. 2013;39:1916-20.

48. Wetterstrand $\mathrm{O}$, Holopainen JM, Krootila K. Treatment of postoperative keratoplasty astigmatism using femtosecond laser-assisted intrastromal relaxing incisions. J Refract Surg. 2013;29:378-82.

49. Loriaut P, Sandali O, El Sanharawi M, Goemaere I, Borderie V, Laroche L. New combined technique of deep intrastromal arcuate keratotomy overlayed by LASIK flap for treatment of high astigmatism. Cornea. 2014;33:1123-8.

50. Geggel HS. Arcuate relaxing incisions guided by corneal topography for postkeratoplasty astigmatism: vector and topographic analysis. Cornea. 2006;25:545-57.

51. Vickers LA, Gupta PK. Femtosecond laser-assisted keratotomy. Curr Opin Ophthalmol. 2016;27:277-84.

52. Krachmer JH, Fenzl RE. Surgical correction of high postkeratoplasty astigmatism. Relaxing incisions vs wedge resection. Arch Ophthalmol. 1980; 98:1400-2.

53. Fadlallah A, Mehanna C, Saragoussi JJ, Chelala E, Amari B, Legeais JM. Safety and efficacy of femtosecond laser-assisted arcuate keratotomy to treat irregular astigmatism after penetrating keratoplasty. J Cataract Refract Surg. 2015:41:1168-75.

54. Cherfan DG, Melki SA. Corneal perforation by an astigmatic keratotomy performed with an optical coherence tomography-guided femtosecond laser. J Cataract Refract Surg. 2014;40:1224-7.

55. Price FW Jr, Whitson WE. The art of surgical correction for postkeratoplasty astigmatism. Int Ophthalmol Clin. 1991;31:59-67.

56. Hjortdal JO, Ehlers N. Paired arcuate keratotomy for congenital and postkeratoplasty astigmatism. Acta Ophthalmol Scand. 1998;76:138-41.

57. Hannush S, Hanna KD, Riveroll L, Gray T, Culbertson WW, Othenin Girard P, et al. Mechanized arcuate keratotomy for astigmatism: a prospective multicenter trial. Invest Ophthalmol Vis Sci. 1998;39:S347. ARVO E-Abstract 1612.

58. Borderie VM, Touzeau O, Chastang PJ, Laroche L. Surgical correction of postkeratoplasty astigmatism with the Hanna arcitome. J Cataract Refract Surg. 1999;25:205-11.

59. Koay PY, McGhee CN, Crawford GJ. Effect of a standard paired arcuate incision and augmentation sutures on postkeratoplasty astigmatism. J Cataract Refract Surg. 2000;26:553-61.

60. Poole TR, Ficker LA. Astigmatic keratotomy for post-keratoplasty astigmatism. J Cataract Refract Surg. 2006;32:1175-9.

61. Hoffart L, Touzeau O, Borderie V, Laroche L. Mechanized astigmatic arcuate keratotomy with the Hanna arcitome for astigmatism after keratoplasty. J Cataract Refract Surg. 2007;33:862-8.

62. Bahar I, Levinger E, Kaiserman I, Sansanayudh W, Rootman DS. IntraLaseenabled astigmatic keratotomy for postkeratoplasty astigmatism. Am J Ophthalmol. 2008;146:897-904.e1.

63. Buzzonetti L, Petrocelli G, Laborante A, Mazzilli E, Gaspari M, Valente P. Arcuate keratotomy for high postoperative keratoplasty astigmatism performed with the IntraLase femtosecond laser. J Refract Surg. 2009; 25(8):709-14.

64. Nubile M, Carpineto P, Lanzini M, Calienno R, Agnifili L, Ciancaglini M, et al. Femtosecond laser arcuate keratotomy for the correction of high astigmatism after keratoplasty. Ophthalmology. 2009;116:1083-92.

65. Kumar NL, Kaiserman I, Shehadeh-Mashor R, Sansanayudh W, Ritenour R, Rootman DS. IntraLase-enabled astigmatic keratotomy for post-keratoplasty astigmatism: on-axis vector analysis. Ophthalmology. 2010;117:1228-35.e1.

66. Fares U, Mokashi AA, Al-Aqaba MA, Otri AM, Miri A, Dua HS. Management of postkeratoplasty astigmatism by paired arcuate incisions with compression sutures. Br J Ophthalmol. 2013;97:438-43.

67. Loriaut $\mathrm{P}$, Borderie VM, Laroche L. Femtosecond-Assisted Arcuate Keratotomy for the Correction of Postkeratoplasty Astigmatism: Vector Analysis and Accuracy of Laser Incisions. Cornea. 2015;34:1063-6.

68. Hashemian M, Ojaghi H, Mohammadpour M, Jabbarvand M, Rahimi F, Abtahi MA, et al. Femtosecond laser arcuate keratotomy for the correction of postkeratoplasty high astigmatism in keratoconus. J Res Med Sci. 2017;22:17.

69. Wu E. Femtosecond-assisted astigmatic keratotomy. Int Ophthalmol Clin. 2011;51:77-85. 